\title{
Structural Safety Assessment of Connection between Sloshing Tank and 6-DOF Platform Using Co-Simulation of Fluid and Multi-Flexible-Body Dynamics
}

\author{
Sang-Moon Yun ${ }^{1}$, Sung-Pill Kim ${ }^{2}$, Soh-Myung Chung ${ }^{1}$, Woo-Jin Shin ${ }^{1}$, Dae-Seung Cho ${ }^{1}[$ and \\ Jong-Chun Park ${ }^{1, *}$ (1) \\ 1 Department of Naval Architecture and Ocean Engineering, Pusan National University, Busan 46241, Korea; \\ ymrb2804@pnu.edu (S.-M.Y.); smchung@pnu.edu (S.-M.C.); jinee0121@pnu.edu (W.-J.S.); \\ daecho@pnu.edu (D.-S.C.) \\ 2 H\&A Common Technology R\&D 1 Team, LG Electronics, Changwon-si 51533, Korea; sungpill.kim@lge.com \\ * Correspondence: jcpark@pnu.edu
}

Received: 18 June 2020; Accepted: 13 July 2020; Published: 24 July 2020

\begin{abstract}
Prediction of sloshing loads, which is one of the most important issues in the design of LNG carriers, has usually been carried out by experiments. When designing a 6-DOF platform equipment used for sloshing experiments, it should target a system containing a fluid, not a solid, thereby making it difficult to predict precisely the dynamic load due to the changes of a center of mass according to the tank's movement. In the present study, two-way co-simulation technology between DualSPHysics and RecurDyn has been developed to analyze the mechanical behavior in multi-body system coupled with fluid motion; in which DualSPHysics is an open-source code based on particle method for fluid analysis and RecurDyn a commercial software for multi-flexible-body dynamics (MFBD). The developed technology was applied to the sloshing problem inside a tank connected to an upper plate on a 6-DOF platform. The simulation results were verified through comparison with the experiments conducted for this study independently, such as snapshots of flow motion, pressure on the cargo hold, and force applied to the tank-platform connection. Finally, to investigate the effects of fluid dynamic load on structural safety assessment, a two-way co-simulation between fluid-MFBD analysis was performed for two cases filling partially with fluid and solid. As a result, it was concluded that the sloshing experiment system used in this study was quite safe, and the feasibility of using the present co-simulation technology for structural safety assessment was confirmed.
\end{abstract}

Keywords: Stewart-Gough platform; sloshing experiments; co-simulation of fluid and multi-flexible-body dynamics; DualSPHysics; RecurDyn; structural safety assessment; multiphysics

\section{Introduction}

The energy issue has been one of the hottest topics since the Industrial Revolution. In particular, petroleum has been taking up the largest share of energy used to date. However, environmental problems such as global warming, extreme weather, and air pollution caused by sulfur oxides from combustion of petroleum are reaching serious levels. As a countermeasure against this, eco-friendly energy policies (i.e., international maritime organization 2020, IMO 2020) are being strengthened worldwide, and the demand for natural gas (NG) fuels, which have relatively superior advantages in terms of environment compared to other fossil fuels, is gradually increasing. However, since natural gas exists in a gaseous state at room temperature, it is inefficient to transport it as it is. Therefore, natural gas is supposed to be loaded 
into a ship's cargo hold in the state of liquefied natural gas (LNG) in a cryogenic environment and to be transported through the sea. This kind of ship is called an LNG tanker or an LNG carrier.

During the operation of LNG ships, they exercise under the influence of various marine environments, and at this time, the relative motion between the internal fluid and the cargo hold occurs, which is called sloshing. In particular, the closer the natural frequency of the fluid and the frequency of the tank motion (or the frequency of the ship motion) get to each other, the more violent the sloshing becomes due to the resonance phenomenon. This can cause structural damage by repeatedly generating instantaneous fluid-induced impact loads on the inner wall of the tank, and it can also affect the global motion of the ship leading to the stability problems during operation [1-4].

Until recently, a lot of researches on sloshing have mainly been conducted through experiments. Akyildiz and Unal [5] conducted a study on the pressure change acting on the wall according to the presence or absence of baffle and various filling ratios through a model test on the sloshing phenomenon inside the square tank. Kishev et al. [6] performed a sloshing experiment according to the filling ratio, sampling rate, and pressure point height of the sidewall to compare and verified the numerical analysis results. Bunnik et al. [7] conducted a model test according to the filling ratio change in a 1:10 ratio size of the prismatic tank of a No96 LNG carrier (Gaztransport \& Technigaz, Saint-Rémy-lès-Chevreuse, France) and studied the characteristics of various sloshing phenomena such as the characteristics of the gas-fluid interaction and hydro-elastic effect. In order to perform the experimental research on the sloshing phenomenon, it is necessary to make the tank move in the same way as the ship's motion, and most experimental studies simulate the ship's motion by utilizing the device that gives the tank excitation. In particular, since an actual ship has a 6-degrees of freedom (DOF) motion when operated, many experimental institutions have been conducting research on the sloshing phenomenon using a 6-DOF device based on the Stewart-Gough platform [8-12].

The 6-DOF platform is widely used not only in shipbuilding and marine industry but also in various applied engineering fields, and many studies have been conducted to design a stable and accurate motion simulator. Stoughton and Arai [13] modified the existing Stewart platform to improve the balance of force, torque, and speed. Wang and Ehmann [14] conducted a study on error analysis methods that can be useful for the precise design of a 6-DOF platform-based device. Moreover, Ding et al. [15] proposed a 6-DOF platform-based device capable of ultra-high accuracy position control even under a large load from the outside and performed stiffness analysis by combining the robot kinematics error model, an analytical method, and the finite element method, a numerical method. However, unlike when a simple rigid body is placed on a platform, if the fluid, located inside the structure, violently moves similar to a sloshing experiment, the center of mass changes over time, thereby making it very difficult to predict the dynamic loads applied to the platform. In addition, researches related on this are still hard to find. Particularly, in the case of connecting parts between the platform and tank, there is a structural risk that of highly possible damage due to concentrated stress. In fact, in March 2011, an impact load corresponding to a wave height of $7 \mathrm{~m}$ was applied to the bottom of the ship due to the tsunami in Japan, and as shown in Figure 1 (Intmarine.com/lnt-a-box-system), a case was reported that the hull of the LNG carrier and the supporting part of the cargo hold were damaged [16]. Therefore, the accurate structural safety assessment technology considering the dynamic load of fluid is essential.

In this study, the co-simulation technique between fluid and multi-flexible body dynamics was developed to perform the structural safety assessment at the connecting parts between the 6-DOF platform and the tank when the rectangular sloshing tank in which the fluid is partially loaded is excited. In order to analyze the fluid whose surface is non-linear and free, the particle-based method from the Lagrangian perspective, which shows strength in fluid impact problems, was adopted instead of the grid-based approach from the Eulerian viewpoint using volume-of-fluid (VOF) [17], level-set [18], etc., which has been mainly used so far. The smoothed particle hydrodynamics (SPH) method [19] or moving particle semi-implicit (MPS) [20] method has been widely used as a particle-based approach, but in this study, DualSPHysics (https://dual.sphysics.org) [21], an open-source code based on weakly 
compressible smoothed particle hydrodynamics (WCSPH), which has the advantage of fast calculation by solving pressure as an explicit method based on the equation of state, was used. On the other hand, the RecurDyn (FunctionBay, Seongnam-si, Rep. of Korea) [22] commercial program that can analyze multi-flexible-body dynamics (MFBD) was used for the analysis of the motion reproduction and the structure of the 6-DOF platform, and the co-simulation technology was developed to link each program. Furthermore, to obtain validation data of the simulation technology, the 6-DOF platform was given a 1-DOF motion in the sway direction to excite a square tank partially filled with water, and the pressure inside the excited tank and the tensile-compression force, applied to the load cell at the connection, was measured. In order to reproduce the same excitation condition as the experiment, with RecurDyn the accurate motion of the top plate of the 6-DOF excluding the fluid was simulated, and then co-simulation with the fluid solver was performed. For the sloshing flow, STAR-CCM+ (Siemens, Munich, Germany) [23], a grid-based commercial computational fluid dynamics (CFD) program, was used for comparison and analysis of the results with those of the particle method. Finally, in order to confirm the effects considering the fluid dynamic load, the structural safety assessments through the finite element analysis (FEA) of the connecting parts were performed and compared for two cases of a solid filler and a fluid filler having the same density and filling ratio.

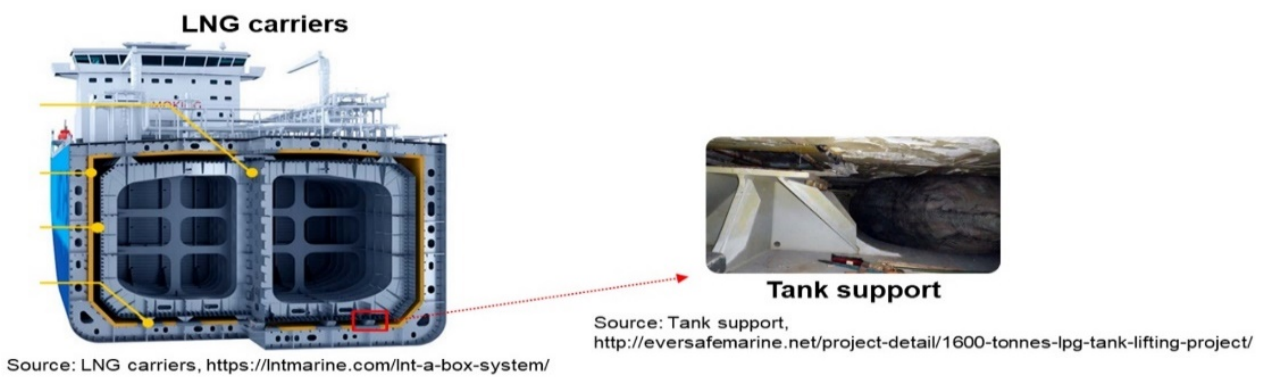

Figure 1. Damaged tank support of cargo hold in LNG carrier [16].

\section{Initial Setup and Condition of Experiments}

As shown in Figure 2, the tank used in the experiment is a rectangular parallelepiped tank scaled down to $1 / 50$ of the second cargo hold of a $138,000 \mathrm{~m}^{3}$ class LNG carrier, and its size $(L \times B \times H)$ is $0.874 \times 0.07 \times 0.535 \mathrm{~m}^{3}$. The material of the tank was made of transparent acrylic with a thickness of $0.02 \mathrm{~m}$ to observe the flow. A 6-DOF platform device of the Global Core Research Center for Ships and Offshore Plants (GCRC-SOP) in Pusan National University (PNU) was used to apply forced motion to the tank [24]. Figure 3 shows an overview of the experimental equipment and a square tank.

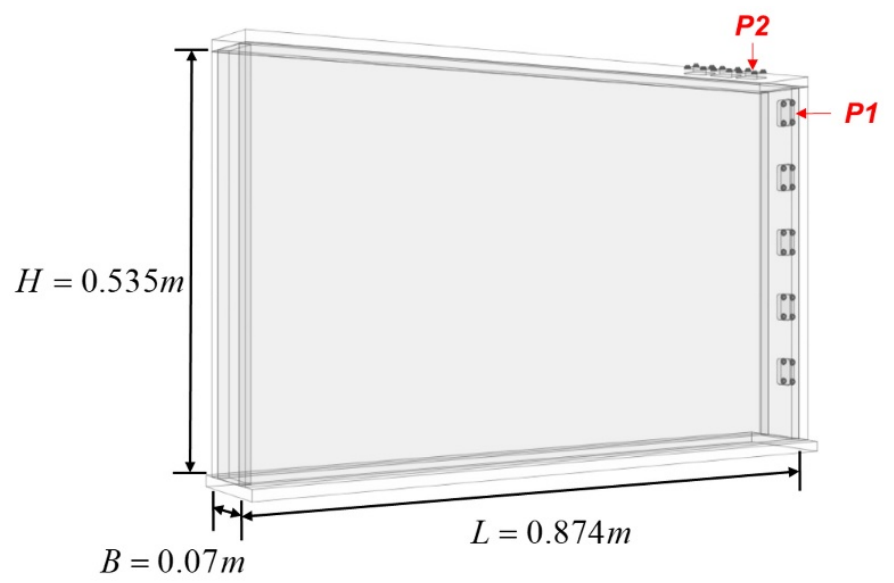

Figure 2. Dimension of rectangular tank. 


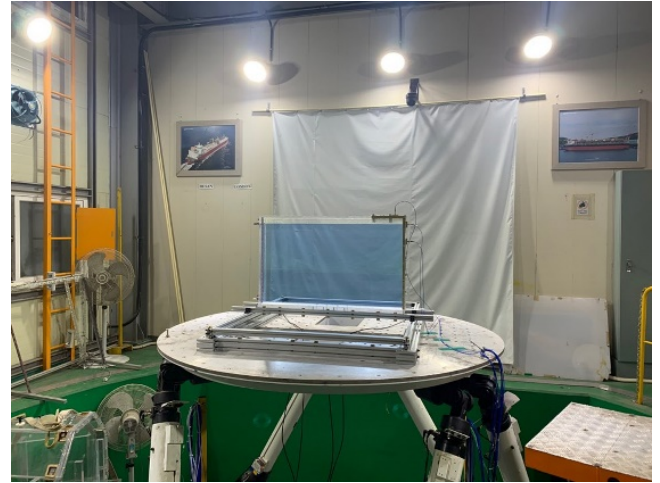

(a)

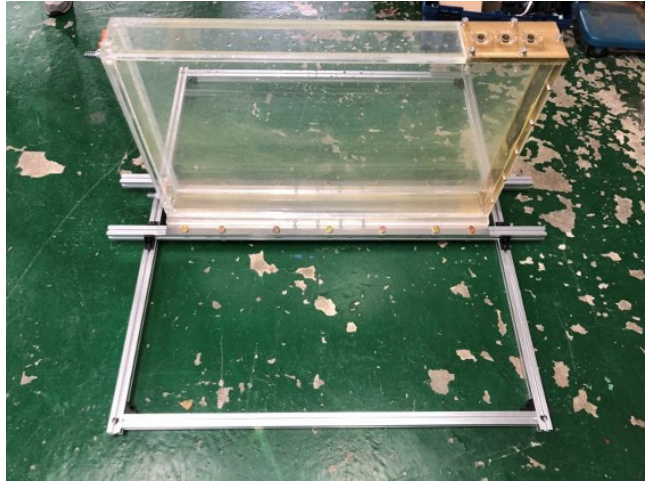

(b)

Figure 3. Sloshing experiment; (a) overview and (b) rectangular tank.

The 1-DOF forced sway movement of the tank can be expressed as Equation (1).

$$
X_{S}=A \sin \left[\left(\frac{2 \pi}{T}\right) t\right]
$$

Here $A$ refers to the amplitude, $T$ the period of motion, and $t$ the time.

In this study, the excitation amplitude was set to $0.087 \mathrm{~m}$, which is $10 \%$ of the tank length. In addition, the filling ratio of the internal fluid of the tank was about $75 \%$, and the excitation period was set to $1.12 \mathrm{~s}$ through Equation (2) used for calculating the natural period according to the filling ratio [25].

$$
T_{n}=\frac{2 \pi}{\sqrt{\frac{\pi}{L} g \tanh \left(\frac{\pi d}{L}\right)}}
$$

where $L$ refers to the length of the tank, $g$ the gravitational acceleration, and $d$ the filling height of the fluid.

The measurement instruments used in the experiment were a high-speed camera for observing the entire sloshing flow, a sensor for measuring the pressure inside the tank, and a load cell for measuring the force applied to the connection between the tank and the 6-DOF platform. Above all, the high-speed camera was the FASTCAM UX 100 model (Photron, Tokyo, Japan) (up to 800,000 fps), and the pressure sensor, the Integrated Circuit Piezoelectric (ICP)-type 211B5 model (Kistler Group, Winterthur, Switzerland) (pressure range $7 \mathrm{bar}$, diameter $5.54 \mathrm{~mm}$, natural frequency $300 \mathrm{kHz}$ ) was used. As shown in Figure 4, a total of two sensors (P1 and P2) were arranged near the upper right corner where the impact load in the rectangular tank mainly occurs. P1 is located on near the right wall $0.5 \mathrm{~m}$ away from the bottom wall, and P2 is located on the upper wall $0.05 \mathrm{~m}$ away from the right wall. Meanwhile, as a load cell, BONGSHIN's DBBP-200 model (Bongshin Loadcell Co., LTD, Osan-si, Korea) (rated capacity $200 \mathrm{kgf}$, sampling rate $20 \mathrm{kHz}$ ) was used. Moreover, as shown in Figure 5, S-beam load cells were placed on the four corners of the jig installed between the sloshing tank and the 6-DOF platform and connected by a bolt fastened method. Through this, the tensile-compression force applied to the connection during the sloshing experiment was measured. 


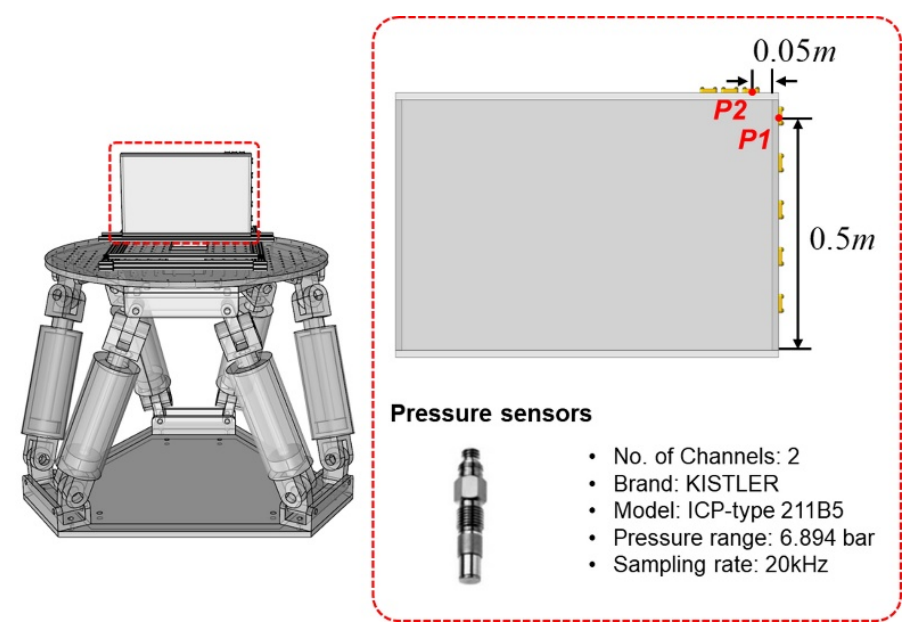

Figure 4. Pressure sensors for experiment.

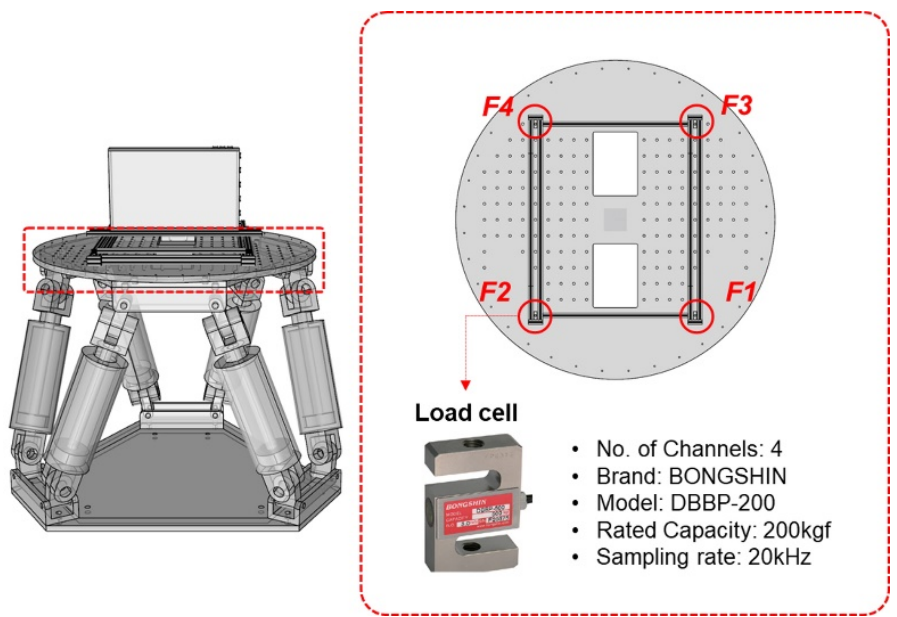

Figure 5. Load cells for experiment.

\section{Numerical Method}

\subsection{Fluid Dynamics Analysis (DualSPHysics)}

The equations governing the behavior of the fluid are the continuity Equation (3) and the momentum Equation (4), and they are as follows.

$$
\begin{gathered}
\frac{D \rho}{D t}=-\rho \frac{\partial v^{\beta}}{\partial x^{\beta}} \\
\frac{D v^{\alpha}}{D t}=\frac{1}{\rho} \frac{\partial \sigma^{\alpha \beta}}{\partial x^{\beta}}+F^{\alpha}
\end{gathered}
$$

where, $\rho$ is the density, $t$ the time, $v$ the velocity, $\alpha$ and $\beta$ the tensors indicating the direction in space, $\sigma$ the total stress, and $F$ the external force.

Equations (3) and (4) can be expressed as the following Equations (5) and (6) by discretization through kernel function and particle approximation [26].

$$
\frac{D \rho_{i}}{D t}=\sum_{j=1}^{N} m_{j}\left(v_{i}^{\beta}-v_{j}^{\beta}\right) \cdot \frac{\partial W_{i j}}{\partial x_{i}^{\beta}}+2 \delta h \sum_{j=1}^{N} m_{j} \bar{c}_{i j}\left(\frac{\rho_{i}}{\rho_{j}}-1\right) \frac{1}{r_{i j}^{2}+\eta^{2}} \cdot \frac{\partial W_{i j}}{\partial x_{i}^{\beta}}
$$




$$
\frac{D v_{i}^{\alpha}}{D t}=-\sum_{j=1}^{N} m_{j}\left(\frac{P_{j}+P_{i}}{\rho_{j} \rho_{i}}+\prod_{i j}\right) \frac{\partial W_{i j}}{\partial x_{i}^{\alpha}}+g_{i}
$$

where $i$ refers to the particle to be calculated, $j$ to the surrounding particle within the effective radius, $P$ to the pressure, $m$ to the mass, $g$ to the gravitational acceleration. Moreover, $c_{i j}=0.5\left(c_{i}+c_{j}\right)$ refers to the sound speed, $\eta^{2}=0.01 h^{2}$ to the coefficient that prevents the denominator from becoming zero and $\mathrm{W}$ to the kernel function in which values are given depending on the distance between particles, and the Quintic kernel [27] was used as the kernel function in this study. $\prod_{i j}$ is a viscous term applied with artificial viscosity, proposed by Monaghan [26]. In addition, for stabilization of pressure, the $\delta$-SPH method proposed by Molteni and Colagrossi [28] was applied to the continuous equation as in Equation (5), and the coefficient $\delta$ was 0.1. Finally, the pressure was calculated through the equation of state as shown in Equation (7) below.

$$
P=B\left[\left(\frac{\rho}{\rho_{0}}\right)^{\gamma}-1\right]
$$

Here, $\gamma=7, B=c_{0}^{2} \rho_{0} / \gamma, \rho_{0}=1000 \mathrm{~kg} \mathrm{~m}^{-3}$ refers to the reference density, and $c_{0}=c\left(\rho_{0}\right)=\sqrt{(\partial P / \partial \rho)} \mid \rho_{0}$ refers to the speed of sound at the reference density.

In this study, the open-source code DualSPHysics based on the SPH method as described above was used for the fluid analysis, and additional details can be found in Crespo et al. [21].

\subsection{Multi-Flexible-Body Dynamics Analysis (RecurDyn)}

First of all, basically, in multi-body dynamics (MBD), it is assumed that the object or system to be analyzed is a rigid body without deformation, and the motion of the rigid bodies is tracked over time. Equations according to position, velocity, and acceleration considering constraints can be expressed as Equations (8)-(10).

$$
\begin{gathered}
\Phi(q, e)=0 \\
\Phi_{u} \dot{u}=-\Phi_{v} \dot{v}-\Phi_{e} \\
\Phi_{q} \ddot{q}=-\left[\left(\Phi_{q} \dot{q}\right)_{q} \dot{q}+2 \Phi_{q e} \dot{q}+\Phi_{e e}\right]
\end{gathered}
$$

Here, $q$ refers to the coordinates of the number of DOF of system, $u$ to the independent coordinates, $e$ to the dependent coordinates, $\Phi$ to the kinematic constraint, and $\Phi_{q}$ to the Jacobian matrix which is the partial derivative of the constraint by the number of coordinates.

By introducing the Lagrange multiplier theory to the constraint equation consisting of algebraic equations, the differential algebraic equation (DAE) that combines the equations of motion consisting of differential equations can be expressed as Equation (11), which becomes the governing equation for the motion of a rigid body when constraints are included.

$$
\left|\begin{array}{cc}
M & \Phi_{q}^{T} \\
\Phi_{q} & 0
\end{array}\right| \begin{gathered}
\ddot{q} \\
\lambda
\end{gathered}|=| \begin{gathered}
Q \\
\theta
\end{gathered} \mid
$$

Here, $\lambda$ refers to the Lagrange multiplier vector, $M$ to the mass matrix, $Q$ to the force vector including the external force, and $\theta$ to the term derived when the second derivative of $\Phi$ with respect to time and is expressed as the third term on the right side of Equation (10).

In particular, in this study, MFBD analysis was performed using RecurDyn V9R2, a commercial program capable of co-simulation between MBD and FEA for structural analysis, and related additional details can be found in FunctionBay's website (www.functionbay.com). 


\subsection{Two-way Co-simulation of Fluid and Multi-Flexible-Body Dynamics}

Figure 6 shows a detailed schematic diagram of each program and coupling method used for two-way co-simulation. For the coupling between two programs, the fluid of DualSPHysics and the multi-rigid-body of RecurDyn exchange interaction forces with each other, and the coupling between the dynamic analysis of the multi-rigid-body and FEA of the multi-flexible body is performed in the RecurDyn.

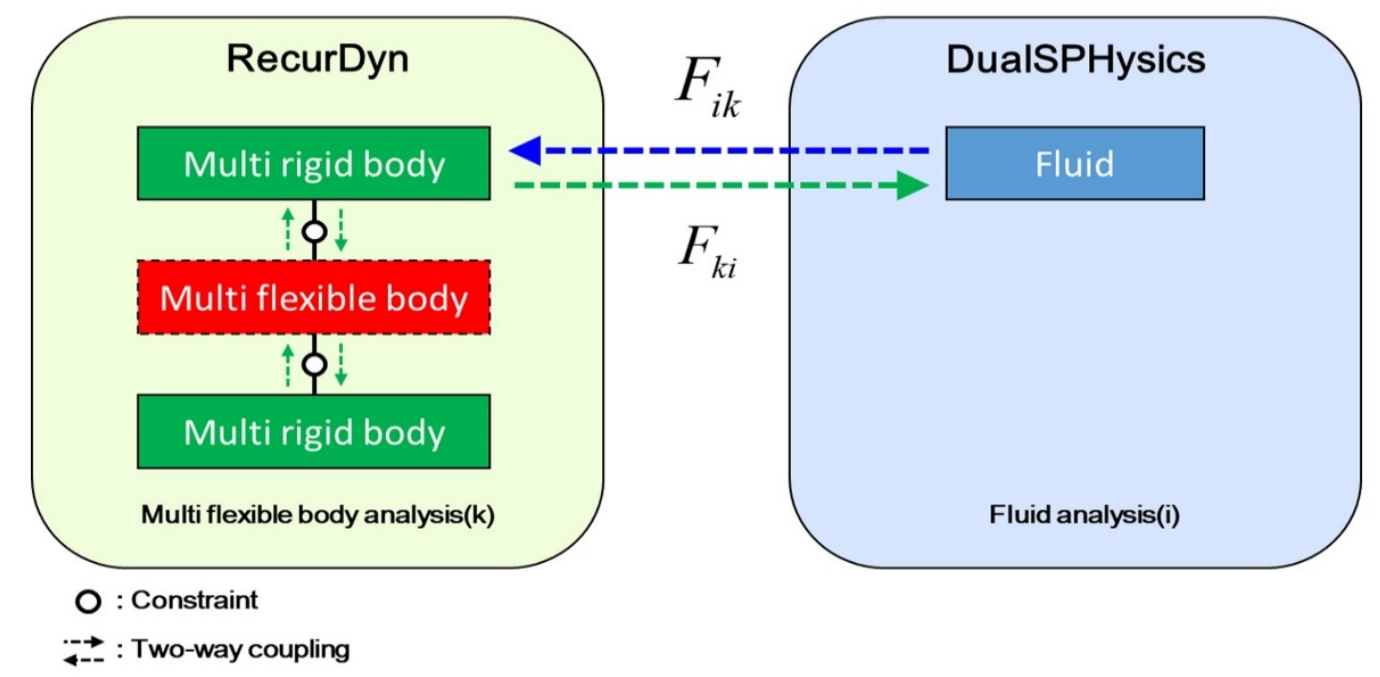

Figure 6. Schematic diagram of two-way coupling method for fluid-MFB dynamics.

In order to define the interaction force for two-way co-simulation between fluid and multi-rigid body, it should be necessary to consider the contact force between the body and the fluid. To this end, in this study, dynamic boundary condition (DBC), proposed by Crespo et al. [29], was applied. The DBC calculates boundary particles in the same equation as fluid particles and is a method that gives repulsive force by increasing pressure as the fluid particles get closer to boundary particles within an effective radius. However, unlike fluid particles, each boundary particle does not move directly, and the entire boundary particle belonging to the body is fixed or moved according to the imposed/assigned motion function.

Finally, as shown in Figure 7, the repulsive force acting on each boundary particle due to the DBC is integrated with respect to the center of gravity to calculate the force and torque $\left(F_{i k}\right)$ acting on the body, and then it is included in the right term of the Equation (11) to perform the MFBD analysis. Moreover, the repulsive force $\left(F_{k i}\right)$ acting on each fluid particle is added to the momentum Equation (6) of the fluid to perform the co-simulation. In this way, the fluid and multi-flexible body exchange interaction forces at every (or constant) time step, and the calculation is performed independently according to each analysis technique. Here, the force exchanged between the fluid and the body particle can be expressed as Equation (12). And by integrating the force applied to each body particle over the entire body, the force $\left(F_{b o d y}\right)$ and torque $\left(\tau_{\text {body }}\right)$ applied to the body can be expressed as Equations (13) and (14).

$$
\begin{gathered}
F_{k i}=-m_{i} \sum_{k=1}^{N} m_{k}\left(\frac{P_{k}+P_{i}}{\rho_{k} \rho_{i}}+\prod_{i k}\right) \nabla_{i} W_{i k}=-F_{i k} \\
F_{b o d y}=\sum_{k=1}^{T} F_{i k} \\
\tau_{b o d y}=\sum_{k=1}^{T}\left(r_{k}-R_{0}\right) \times F_{i k}
\end{gathered}
$$


Here, $i$ refers to the fluid particle, $k$ to the body particle, $T$ to the total number of each body particle, $N$ to the number of neighbor body particle, $R_{0}$ to the center of mass, $r_{k}$ to the position of body particle.

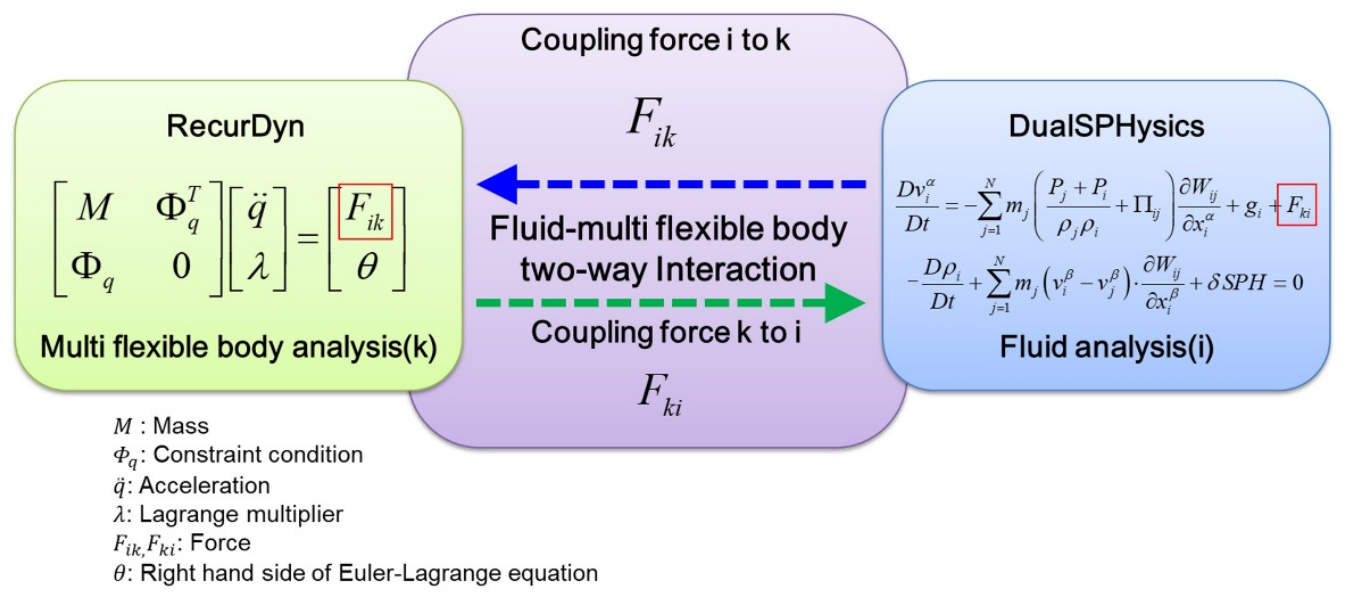

Figure 7. Outline of two-way co-simulation for fluid-MFB dynamics.

The detailed time integration coupling method is shown in Figure 8. In step 1, particle-to-wall forces $\left(F_{i k}\right)$ calculated from $t_{-1}$ to $t_{0}$ act constantly on the individual body of the RecurDyn during $t_{0} \sim t_{1}$. In step 2, RecurDyn passes wall-to-particle force $\left(F_{k i}\right)$ calculated by each body motion information (e.g., position and velocity) during step 1 to DualSPHsics. In step 3, DualSPHysics conducts fluid analysis based on the updated wall information received from RecurDyn. Here, the time increment for fluid analysis in DualSPHysics is set smaller than that for RecurDyn, and several time steps are calculated depending on the time integration scheme of DualSPHysics until just before the time $t_{1}$ in RecurDyn. In addition, the force $\left(F_{i k}^{t_{0} \sim t_{1}}\right)$ from $t_{0}$ to $t_{1}$ is calculated as the time-weighted average as given in the following equation:

$$
F_{i k}^{t_{0} \sim t_{1}}=\frac{1}{\sum_{n=1} \Delta t^{n}} \sum_{n=1} F_{i k}^{n} \Delta t^{n}
$$

Here, $\Delta t^{n}$ implies the time increment in DualSPHysics, $n$ the time step in DualSPHysics, $F_{i k}^{n}$ the particle-to-wall forces at the end of time step $n$ in DualSPHysics.

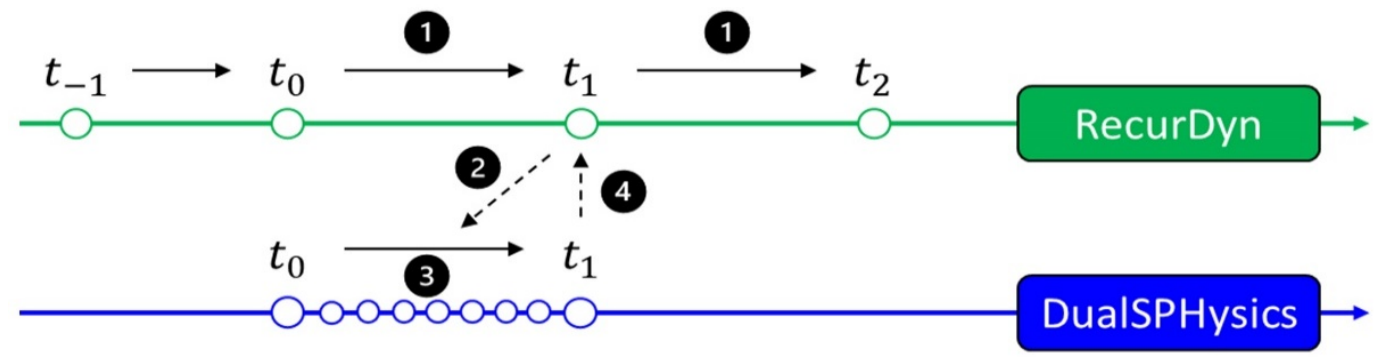

Figure 8. Two-way time step coupling strategy fluid-MFB dynamics.

Lastly, in step 4 , the newly updated forces for $t_{0} \sim t_{1}$ is returned to RecurDyn. These forces act constantly on the corresponding bodies of RecurDyn during $t_{1} \sim t_{2}$ as in step 1 .

As described above, two-way coupling sequential time integration in which steps 1 to 4 are repeated was performed. 


\section{Numerical Results}

\subsection{Validation}

Before performing the structural analysis of the connection between the sloshing tank and the upper plate of the 6-DOF platform, the Fluid-MFB co-simulation was performed to validate the interaction technology between the fluid and the MBD. As shown in Figure 9, the 6-DOF platform and sloshing tank were modeled to have the same conditions as the experiment as much as possible. However, in the case of 6-DOF platform modeling, an arbitrary platform [30] that differs somewhat from the experimental shape was used because detailed specifications were not provided by the manufacturer. In order to reproduce Equation (1) regarding the motion of the tank, the mechanical motion of each of the six legs of the platform was adjusted using inverse kinematics. As a result of measuring the position change in the sway direction from the center point of the upper plate of the platform over time, as shown in Figure 10, it was found that the periodic motion of the tank with an amplitude of $0.087 \mathrm{~m}$ and a period of $1.12 \mathrm{~s}$ was successfully reproduced. However, in order to stabilize the fluid analysis based on the particle method at the beginning, the motion was started after stopping for $1 \mathrm{~s}$.

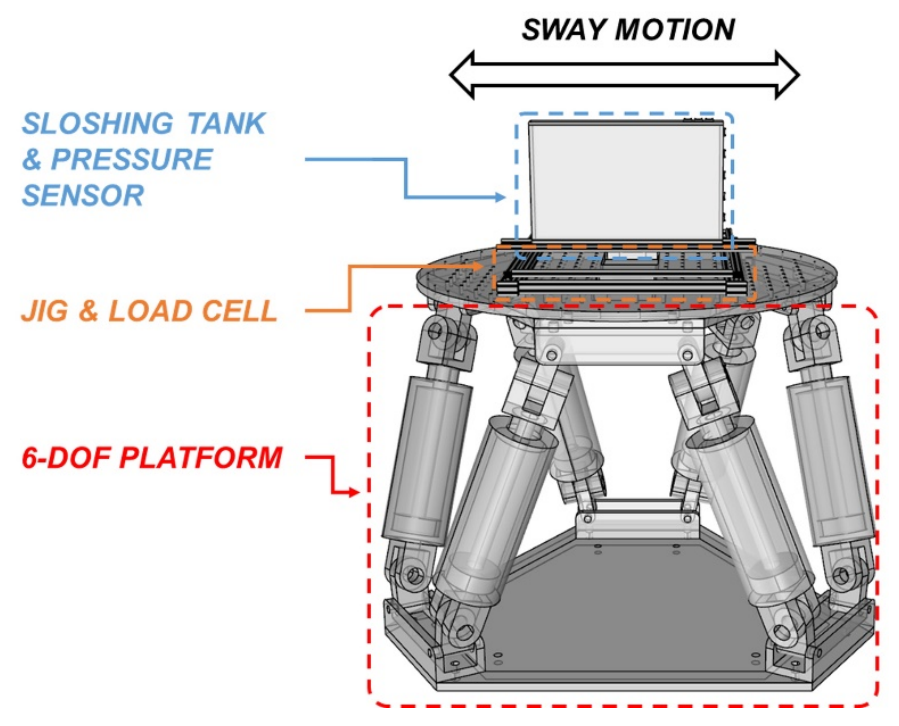

Figure 9. Schematic view of sloshing tank equipped on 6-DOF platform.

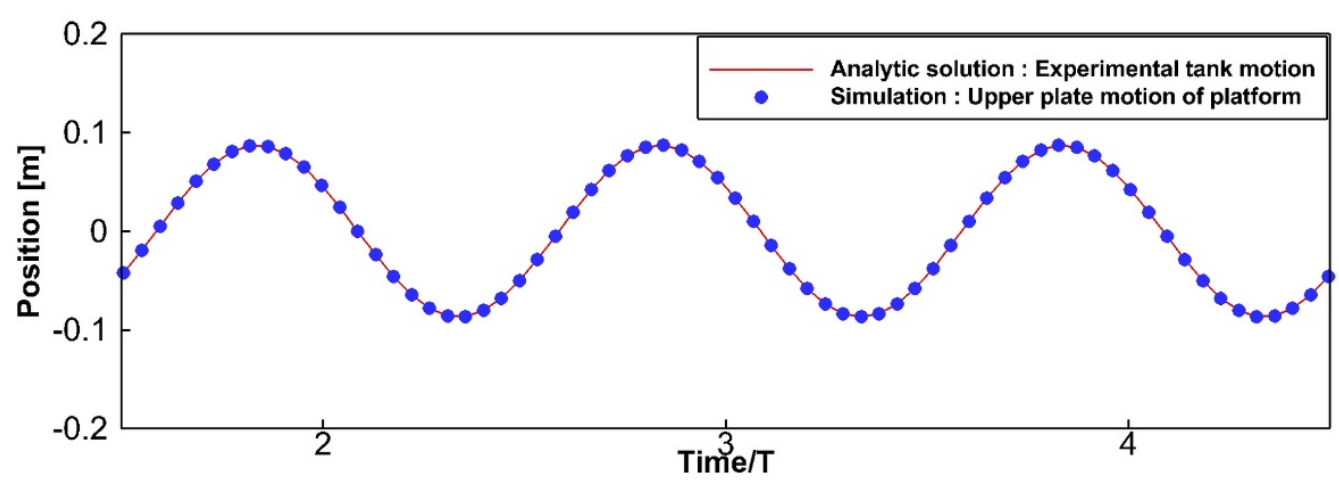

Figure 10. Reproduction of tank motion by 6-DOF platform.

The simulation conditions of DualSPHysics for the fluid analysis are shown in Table 1, and for the validation of simulation results, pressure and force were measured at the same location as the experiment. However, in the case of the force, the average values of F1 and F3, which are symmetrical in direction of the breadth (B) of the tank, were used in the analysis among the results measured in 
four places of F1 to F4. In the convergence test for particle size, 37,000, 370,000, and 3,000,000 particles were used, respectively, with particle resolution of $\Delta x=8 \mathrm{~mm}, 4 \mathrm{~mm}$, and $2 \mathrm{~mm}$, and time-series comparison and convergence results of forces (average of F1 and F3) are shown in Figure 11. As the particle size decreases, a tendency to converge appears, and it can be clearly seen from Figure 11b, which is a result of comparing the relative difference with respect to the maximum value of the force based on the smallest particle size of $2 \mathrm{~mm}$. Therefore, the flow analysis for the validation through comparison with the experiment and for the stress analysis of the connection was performed with $2 \mathrm{~mm}$, the converged particle size.

Table 1. Parameters of DualSPHysics for fluid analysis.

\begin{tabular}{cc}
\hline Parameter & Value \\
\hline Time integration & Symplectic scheme \\
Kernel function & Wendland \\
Density diffusion & Delta-SPH $(\delta=0.1)$ \\
Courant number & 0.2 \\
$h / \Delta x$ (in 3D) & 1.385 \\
Viscosity model & Artificial viscosity $(\alpha=0.01)$ \\
Fluid density & $1000 \mathrm{~kg} / \mathrm{m}^{3}$ \\
\hline
\end{tabular}

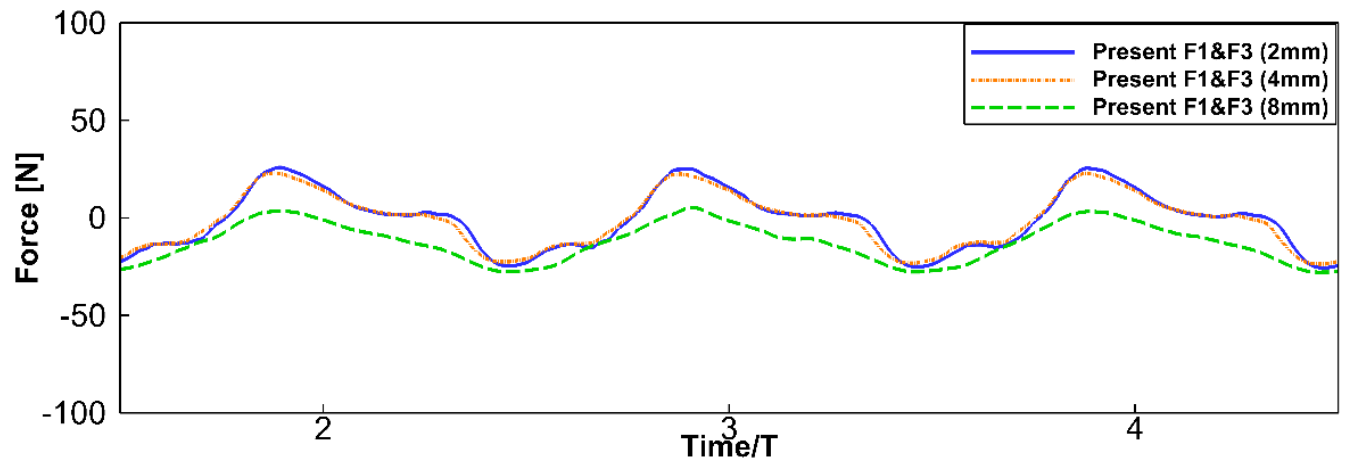

(a)

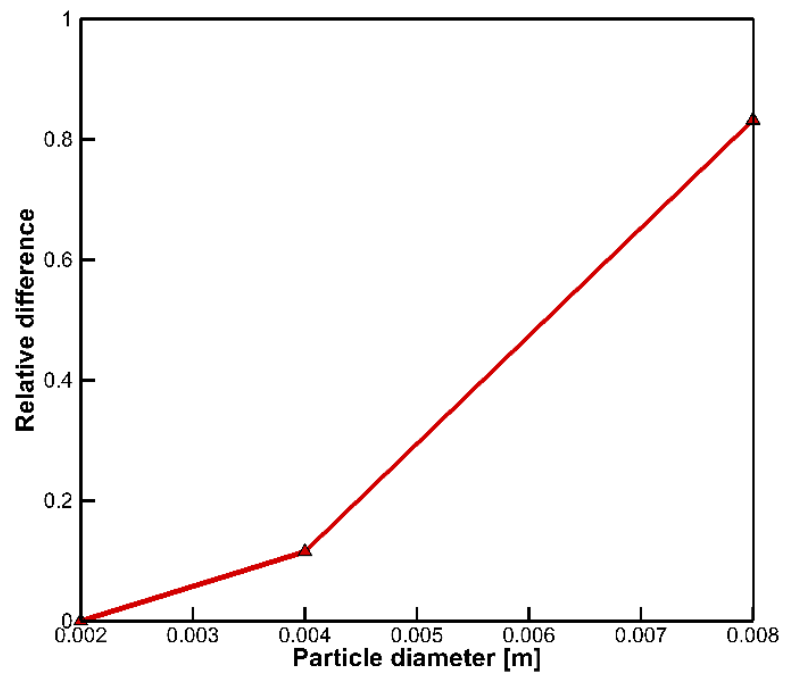

(b)

Figure 11. Convergence test of particle size based on force results; (a) time-series of force and (b) relative difference.

The same two-dimensional sloshing problem was simulated using the grid-based commercial CFD software, STAR-CCM+ version 15.02, for a more precise comparative investigation of flow analysis 
using a particle-based method. However, in the case of STAR-CCM+, co-simulation with RecurDyn cannot be activated at this time, so it was applied only to the sloshing flow problem. As a grid system, as shown in Figure 12, about 300,000 meshes were initially generated with a minimum grid size of $2 \mathrm{~mm}$, and a VOF model for 2-phase flow and a function of adaptive mesh refinement (AMR) were used for effective capturing the free-surface motion with time. Figure 13 shows the comparison of the snapshots for the process of the fluid colliding with the upper right wall in three steps (A) (C) in the experiment and corresponding simulation results. In the case of step $(\mathbb{A}$, the flow was overturned with a collision near the right corner, and the spray following the overturning was better represented by the particle method DualSPHysics. However, for the shape of the free-surface near the left wall, the results of STAR-CCM+ appeared a little more similar to that of the experiment. In the case of step (B), after the collision, the jet flow was sprayed toward the left wall along the upper wall, and DualSPHysics showed an intense and concentrated jet flow compared to the experiment, whereas STAR-CCM+, similar to the result of step (A), which showed that the spray was not properly simulated. In step (B), the pressure distribution of the two simulations was different, especially in the upper right corner. The flow from step (B) to step (C) was observed to gradually move along the upper wall and right side. However, in the case of DualSPHysics, unlike the experiment or STAR-CCM+, the water level rapidly decreased from the upper right wall. This seems to be caused by an insufficient acceleration in the horizontal direction because the negative pressure appearing near the upper right corner of the STAR-CCM+ in step (B) was not accurately represented. In order to fix this problem, further studies such as the improvement of negative pressure treatment and the two-phase flow simulation should be required.

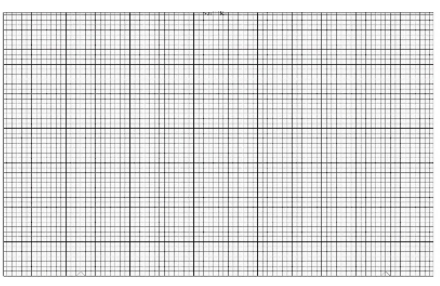

(a)

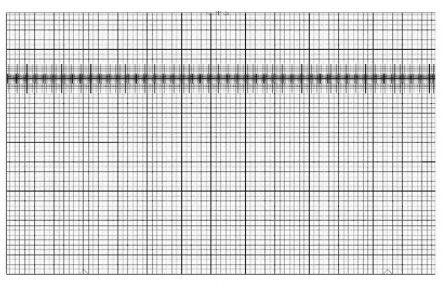

(b)

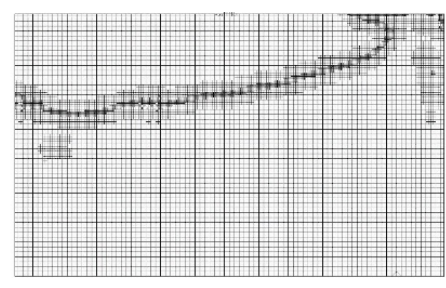

(c)

Figure 12. Grid generation of STAR-CCM+ using AMR; (a) without AMR, (b) with AMR (time/T = 0) and (c) with AMR (time/T = 1.87).

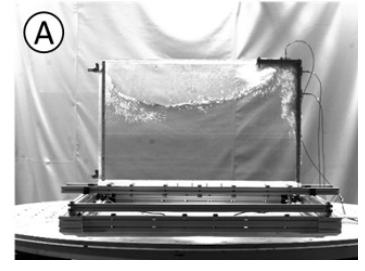

(a)

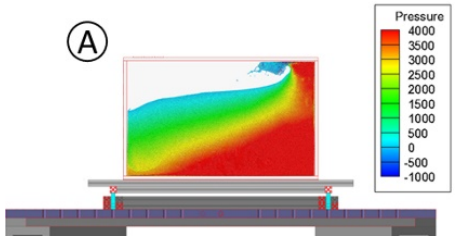

(d)

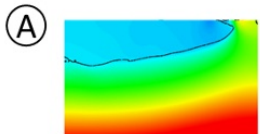

(g)

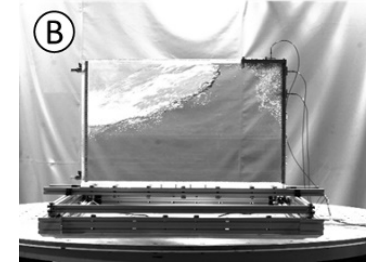

(b)

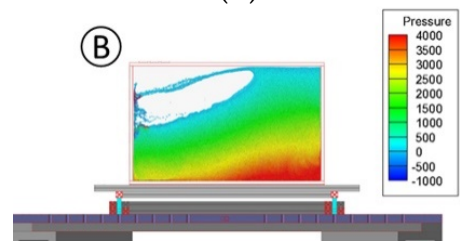

(e)

(B)

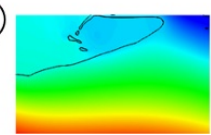

(h)

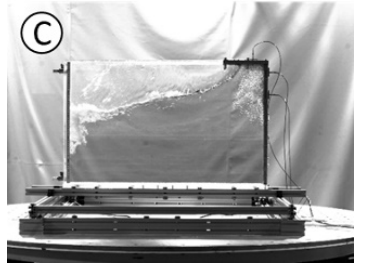

(c)

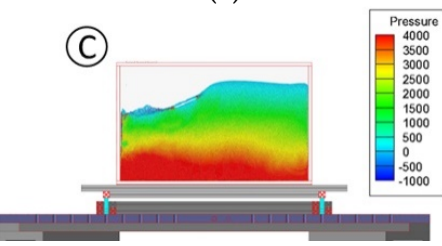

(f)

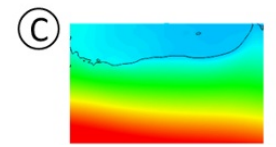

(i)

Figure 13. Comparison of snapshots (pressure contour); (a) exp., time/ $\mathrm{T}=1.87$, (b) exp., time/ $\mathrm{T}=2.05$, (c) exp., time/T = 2.22, (d) present, step $(A)$, (e) present, step B), (f) present, step $\mathbb{O}$, (g) STAR-CCM+, step (A), (h) STAR-CCM+, step (B) and (i) STAR-CCM+, step (O). 
Figure 14 shows the time series data measured by two pressure sensors (P1 and P2) in the tank compared to the experiment and STAR-CCM+. Here, a Butterworth second-order bandpass digital filter (cutoff frequency: $0.001 \mathrm{~Hz}$ to $300 \mathrm{~Hz}$ ) was applied to the pressure time series data. Overall, the period of the peak is similar to the experiment but shows a difference in magnitude. In particular, relative errors of the average magnitude of the peaks at points $\mathrm{P} 1$ and P2, compared to the experiments, were calculated as $14.8 \%$ and $85.8 \%$ for DualSPHysics, and $67.8 \%$ and $60.2 \%$ for STAR-CCM+, respectively. Thus, it can be found that the number of errors in this study was good in P1, but over-estimated in P2. In addition, it can be seen that the negative pressure occurs similar to the experiment in STAR-CCM+ as shown in Figure 13.

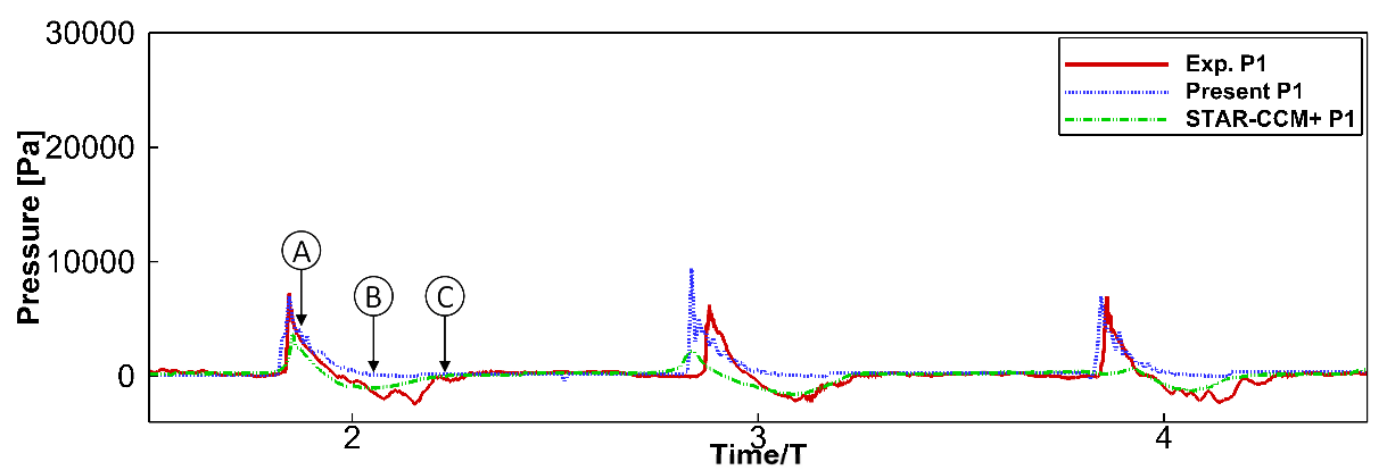

(a)

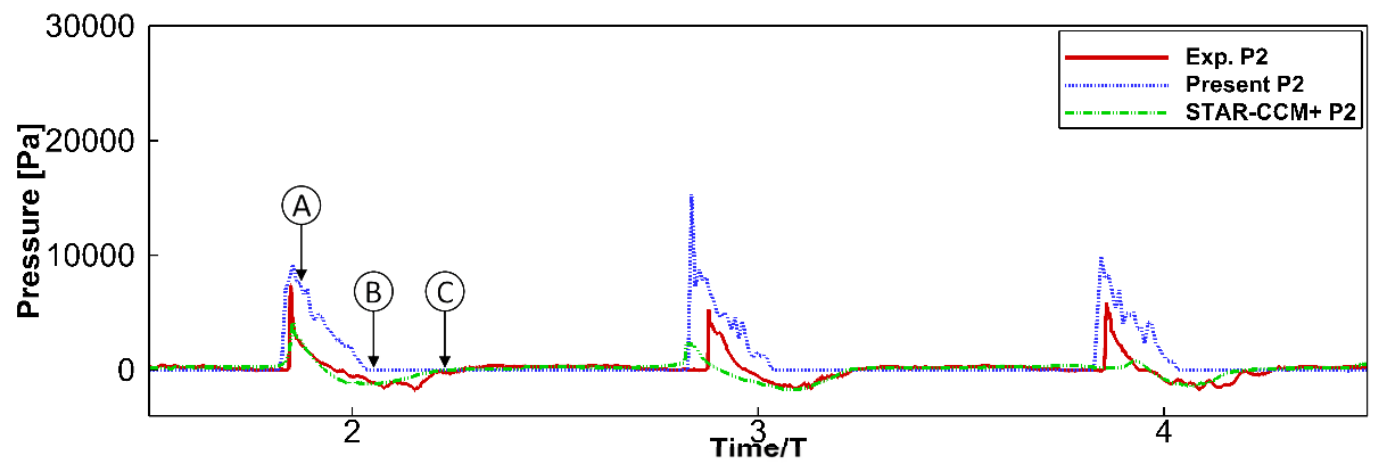

(b)

Figure 14. Comparison of pressure with experiment; (a) pressure sensor P1 and (b) pressure sensor P2.

Since for now, the results of the co-simulation of the flow field obtained by DualSPHysics with MFBD will be discussed. Therefore, any comparison with the flow motion by STAR-CCM+ is no longer discussed. Figure 15 shows the averaged forces on the F1 and F3 measured from the S-beam load cells compared to the experiment, in which a Butterworth 2nd-order bandpass digital filter (cutoff frequency: $0.005 \mathrm{~Hz}$ to $4 \mathrm{~Hz}$ ) was applied to the time-series data of the force. Overall, the period was similar to that of the experiment, and the relative error of the maximum value at the peak was also calculated to be about $11.1 \%$, which approached the experiment. However, in the detailed trend, unlike the experiment, a secondary peak occurs around step $(0$. As pointed out above, this can be analyzed as the influence of the load transferred directly in the direction of gravity due to the absence of negative pressure at the upper ceiling corner in steps (B) and (C) of Figure 13. 


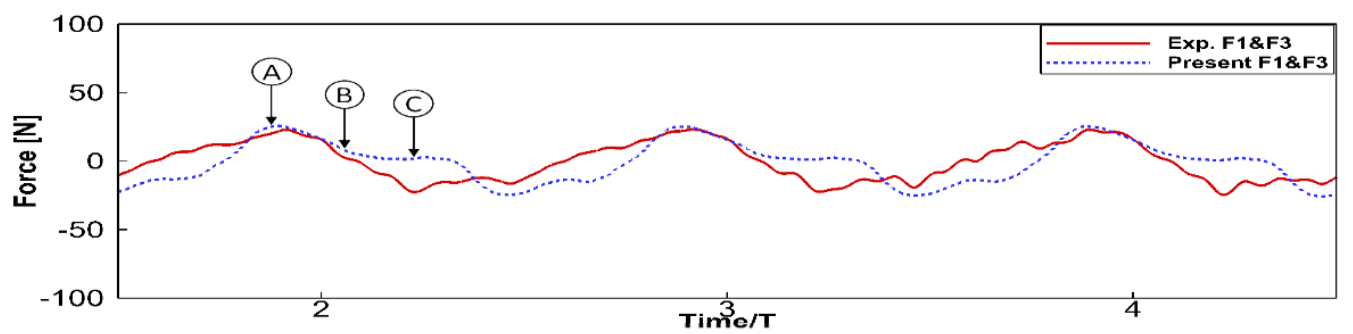

Figure 15. Comparison of average force (F1 and F3) with experiment.

\subsection{Structural Safety Assessment Considering Fluid Dynamic Load}

To evaluate the structural safety of the connection between the tank and the 6-DOF platform, structural stress analysis on the load cell installed at the connection was performed. On this occasion, in order to investigate the influence of the fluid dynamic load, a case of a solid filler having the same density and filling ratio as the fluid was performed and compared. Here, the detailed schematic diagram of the two-way co-simulation for the fluid filler is shown in Figure 16. In the case of the solid fillers, no fluid analysis should be required, so all calculations are done within RecurDyn. In particular, the fluid of DuaSPHysics and the S-beam load cell of RecurDyn interact with each other through a sloshing tank and jig, which are rigid bodies. Since the load cell is strongly connected to the Jig and the 6-DOF platform through the bushing force constraint, it can be regarded as a nearly fixed boundary condition.

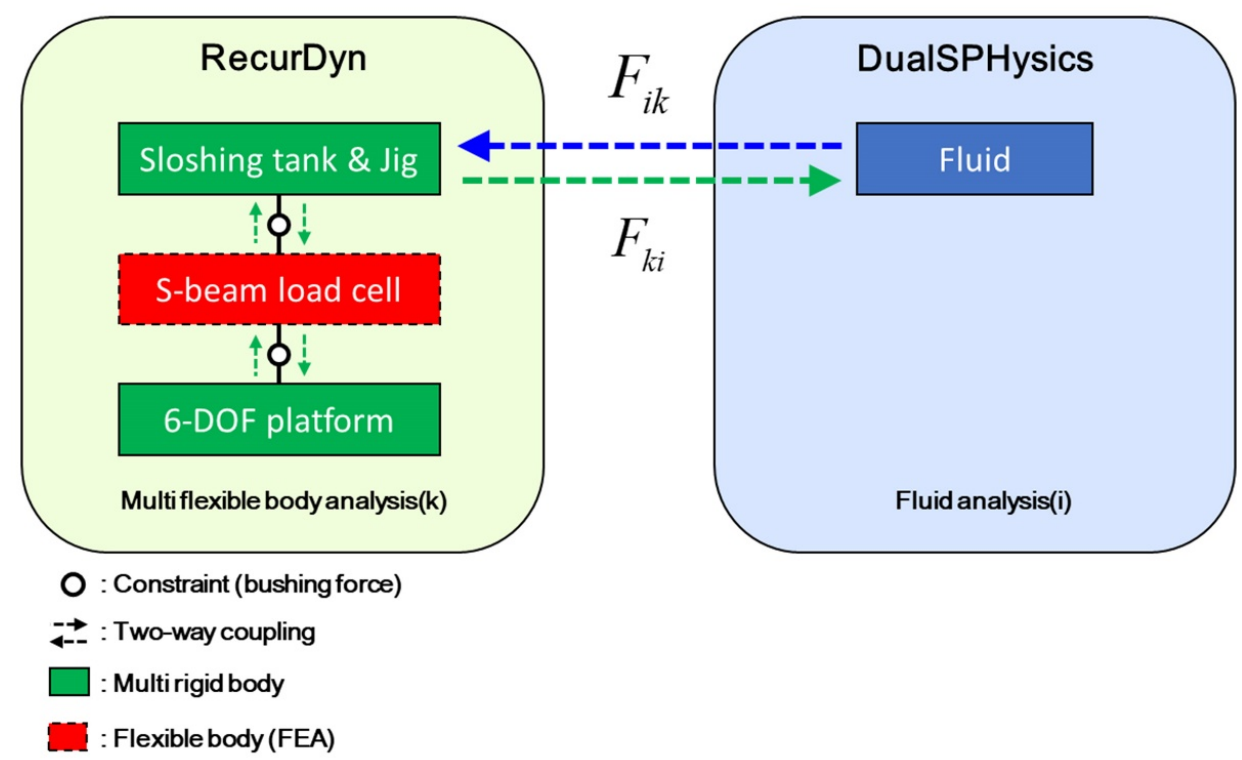

Figure 16. Diagram of two-way co-simulation for fluid filler.

As shown in Figure 17, structural analysis (FEA) was performed using meshes corresponding to 19,135 nodes and 93,791 elements for the S-beam load cell installed at the connecting parts. Here, as given in Table 2, the material properties of 304 stainless steel [31] were used.

Table 2. Mechanical material properties of load cell.

\begin{tabular}{cc}
\hline Property & Value \\
\hline Density $\left(\mathrm{kg} / \mathrm{m}^{3}\right)$ & 7900 \\
Young's modulus $(\mathrm{MPa})$ & 198,500 \\
Poisson's ratio & 0.294 \\
Tensile strength, yield $(\mathrm{MPa})$ & 215 \\
Tensile strength, ultimate $(\mathrm{MPa})$ & 505 \\
\hline
\end{tabular}




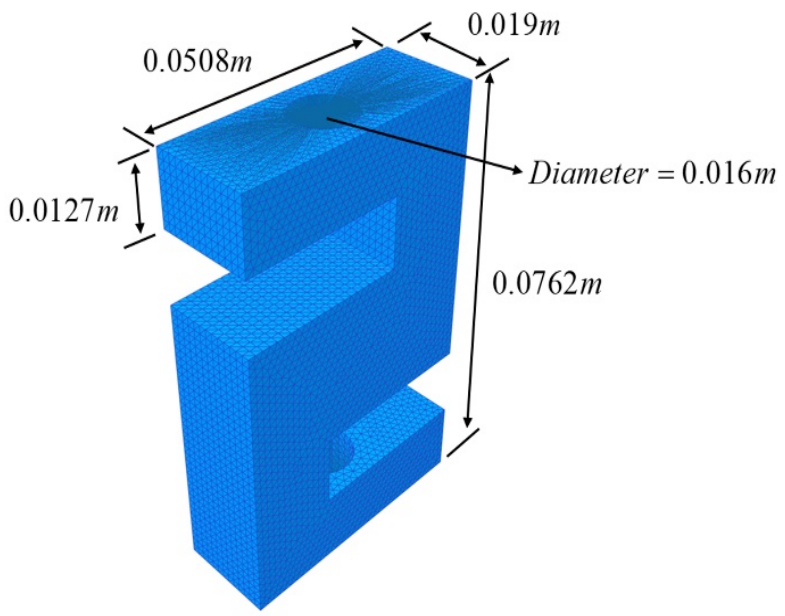

Figure 17. Dimensions and mesh system of S-beam load cell for FEA.

Figure 18 indicates an instantaneous snapshot when the maximum stress appears after the sloshing flow develops under the same excitation conditions as in Figure 10, which was periodically observed near the moment when the excitation is the maximum (acceleration is the maximum). In particular, the dimensionless time at which the maximum stress appears in the fluid case was delayed by about $0.71 \%$, compared to that of the solid case. Figures 19 and 20 show the stress distribution acting on the cross-section of the S-beam load cell when the maximum stress occurs and the time series of the von Mises stress at the location where the maximum stress occurs, respectively. In the stress contour $(0.001 \mathrm{MPa} 5.3 \mathrm{MPa})$, the stress concentration occurred in the vicinity of the two edges, resulting in a large stress compared to the averaged stress of the entire load cell. It can be seen that larger maximum stress occurred repeatedly due to sloshing flow in the fluid tank compared to the solid tank. The maximum stress occurred at the same location, and on average the magnitude of peak stress was $5.39 \mathrm{MPa}$ in the solid tank and $6.50 \mathrm{MPa}$ in the fluid tank, which was measured about $21 \%$ larger in the fluid tank by fluid dynamic loads such as sloshing. As a result of comparing the maximum stress shown above with the yield strength to perform a structural safety assessment of the connection, the solid and fluid tanks can be evaluated to be very safe at about $2.5 \%$ and $3 \%$, respectively. However, if the system is designed using a material with a lower yield strength than the material of the connection used in the sloshing experiment of this study, the possibility of fatigue fracture of the connection may be faster due to repeated fluid dynamic loads.

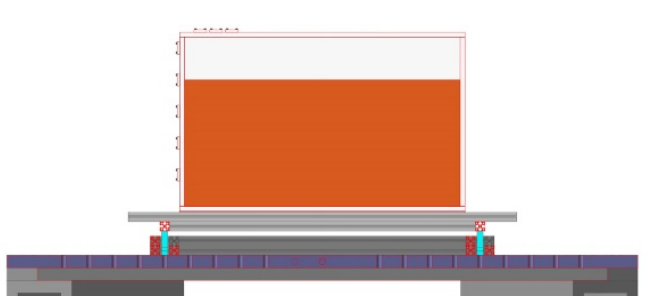

(a)

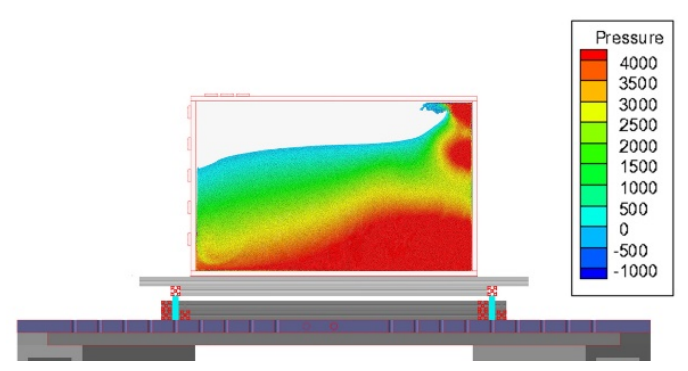

(b)

Figure 18. Comparison of snapshots at the moment of maximum stress; (a) solid case (time/ $\mathrm{T}=1.836$ ) and $(\mathbf{b})$ fluid case (time/ $\mathrm{T}=1.849$ ). 


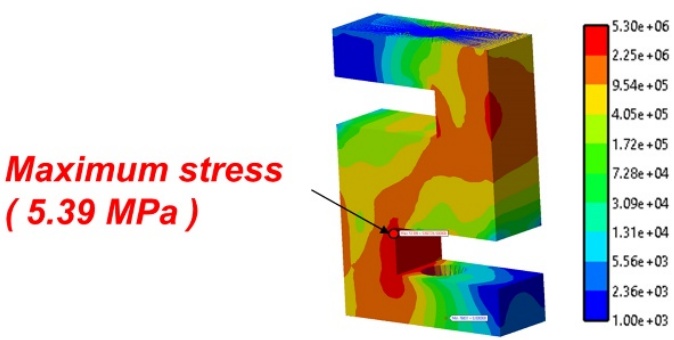

(a)

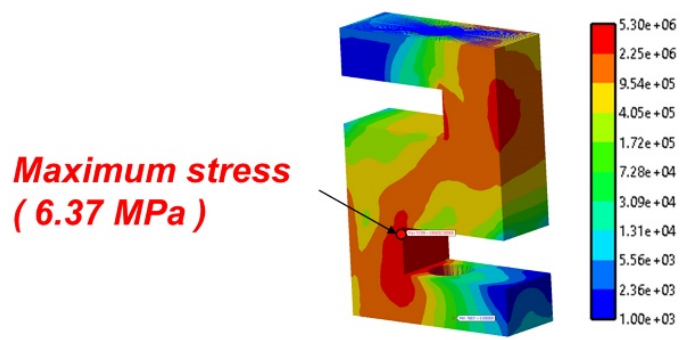

(b)

Figure 19. Comparison of stress contour at the moment of maximum stress; $(\mathbf{a})$ solid case $($ time/ $\mathrm{T}=$ 1.836) and (b) fluid case (time/ $\mathrm{T}=1.849$ ).

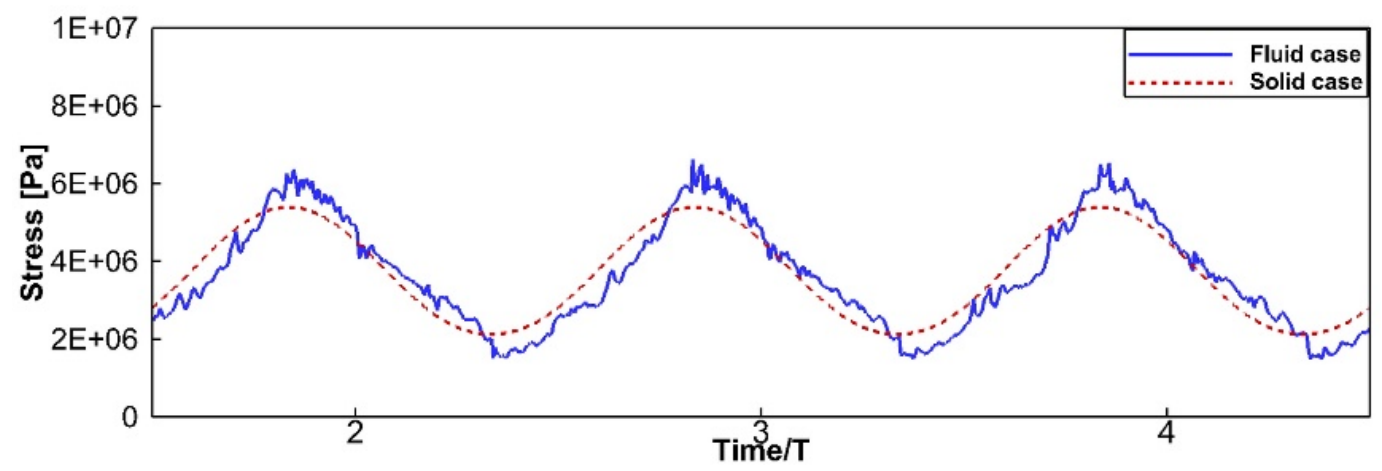

Figure 20. Comparison of von Mises stress.

\section{Conclusions}

In this study, the co-simulation technology between DualSPHysics, an open-source code based on particle method for fluid analysis, and RecurDyn, a commercial software for MFBD, was developed to perform the structural safety assessment of the connection between the sloshing tank and the 6-DOF platform. To validate the co-simulation technique, a sloshing experiment with a simple 1-DOF sway motion was performed. In addition, simulations under the same conditions as in the experiment were performed, and the flow snapshot, the pressure in the tank, and the tensile-compression force acting on the connection between the tank and the 6-DOF platform were compared and analyzed.

In the case of flow analysis, a particle size convergence test was performed, and the converged results were compared with the results of the experiment and grid-based commercial software STAR-CCM+. The results of the comparison are summarized as follows:

- As a result of analyzing the process in which the sloshing flow collides with the upper wall and falls, the spray phenomenon caused by the collision was better expressed in the particle method DualSPHysics. However, the behavior after the collision showed a rapid drop in water level, unlike the experiment and grid-based methods. This seems to be a problem caused by a lack of acceleration in the horizontal direction because the negative pressure was not accurately described by the single-phase particle method.

- In case of the pressure in the tank, the period of the peak was similar to that of the experiment, but the magnitude tends to show overestimation for the particle method and underestimation for the grid method.

- In case of the average of the force (F1 and F3) of the connection, the tendency of the secondary peak that occurs due to the rapid drop in the water level after the collision with the upper wall of the tank was somewhat different from the experiment. However, the average value of the peak showed a relative error of $11.10 \%$ compared to the experiment.

- In the overall validation process, it can be seen that the main parameter resulted in simulation inaccuracy is the pressure appearing negative. Particularly, in the current sloshing experiment 
system, although the influence of pressure by sloshing impact was not enough on the magnitude of the force acting on to the connection, the flow pattern changed due to the incorrect reproduction of negative pressure, and there exists a possibility that it may lead to inaccuracy in the structural safety assessment of other systems.

In order to verify the effect of fluid dynamic load on the structural safety assessment of the connection, the same simulation was performed using a solid tank having the same density and filling ratio as the fluid tank, and the stress on the connection and the snapshots were compared. The results of the structural safety assessment are summarized as follows:

- The maximum stress occurred near the moment when the excitation of the tank was generated at its maximum, and the fluid case showed a delay of about $0.71 \%$ in time with respect to the motion period compared to the solid case.

- The maximum stress occurred in both cases at the same location, and as a result of comparing the maximum stress with yield strength for safety assessment, it was confirmed that both cases were very safe within $3 \%$. However, in the fluid case, the maximum stress of about $21 \%$ greater than that of the solid case was measured. Thus, if other systems are designed using a material with a lower yield strength than the material of the connection used in the sloshing experiment of this study, the possibility of fatigue fracture of the connection may be faster due to repeated fluid dynamic loads. In addition, the equipment used in this study, such as the 6-DOF platform and load cell, corresponds to the actual size. However, it could be said that the simulation result may have been obtained very safely since the sloshing tank was scaled down to $1 / 50$ of its actual size. Therefore, it is necessary to perform a structural safety assessment for actual engineering problems involving larger fluid dynamic loads.

The developed two-way co-simulation technology in this study can be said to have the potential to solve many challenging problems, such as deformation of multi-body interacting with very complex free-surface flow associated with wave-breaking. Therefore, if the problems pointed out through this study are supplemented and verified $[29,32,33]$, i.e., introduction of multi-phase flow for correct implementation of negative pressure and improvement of boundary conditions for complex geometry, it is expected to be used in various engineering fields concern with practical multi-physics.

Author Contributions: Conceptualization, S.-P.K. and J.-C.P.; writing-original draft preparation, S.-M.Y. and J.-C.P.; writing-review and editing, S.-M.Y. and J.-C.P.; software, S.-M.Y. and S.-P.K.; validation, S.-M.Y. and S.-M.C.; visualization, S.-M.Y.; data curation, S.-M.C. and W.-J.S.; supervision, J.-C.P.; project administration, J.-C.P.; funding acquisition, D.-S.C. All authors have read and agreed to the published version of the manuscript.

Funding: This work was supported by the National Research Foundation of Korea (NRF) grant funded by the Korea government(MSIT) through GCRC-SOP (No. 2011-0030013).

Acknowledgments: Special thanks to Gyumok Jeon for providing analysis data of STAR-CCM+, and FunctionBay for permission to use the license of RecurDyn.

Conflicts of Interest: The authors declare no conflict of interest.

\section{References}

1. Peric, M.; Zorn, T.; Moctar, O.; Schellin, T.E.; Kim, Y.-S. Simulation of sloshing in LNG-tanks. J. Offshore Mech. Arct. 2009, 131. [CrossRef]

2. Krata, P. The impact of sloshing liquids on ship stability for various dimensions of partly filled tanks. TransNav Int. J. Mar. Navig. Saf. Sea Transp. 2013, 7, 481-489. [CrossRef]

3. Hu, Z.-Q.; Wang, S.-Y.; Chen, G.; Chai, S.-H.; Jin, Y.-T. The effects of LNG-tank sloshing on the global motions of FLNG system. Int. J. Nav. Arch. Ocean 2017, 9, 114-125. [CrossRef]

4. Kim, S.-P.; Chung, S.-M.; Shin, W.-J.; Cho, D.-S.; Park, J.-C. Experimental study on sloshing reduction effects of baffles linked to a spring system. Ocean Eng. 2018, 170, 136-147. [CrossRef]

5. Akyildiz, H.; Unal, E. Experimental investigation of pressure distribution on a rectangular tank due to the liquid sloshing. Ocean Eng. 2005, 32, 1503-1516. [CrossRef] 
6. Kishev, Z.R.; Hu, C.; Kashiwagi, M. Numerical simulation of violent sloshing by a CIP-based method. J. Mar. Sci. Tech. 2006, 11, 111-122. [CrossRef]

7. Bunnik, T.; Huijsmans, R. Large scale LNG sloshing model tests. In Proceedings of the Seventeenth International Offshore and Polar Engineering Conference, Lisbon, Portugal, 1-6 July 2007.

8. Stewart, D. A platform with six degrees of freedom. In Proceedings of the Institute of Mechanical Engineering, London, UK, 1 June 1965.

9. Ahn, Y.J.; Kim, S.-Y.; Kim, K.-H.; Lee, S.-W.; Kim, Y.H.; Park, J.-J. Study on the effect of density ratio of liquid and gas in sloshing experiment. In Proceedings of the Twenty-second International Offshore and Polar Engineering Conference, Rhodes, Greece, 17-22 June 2012.

10. Kim, S.-Y.; Kim, K.-H.; Kim, Y.H. Comparative study on pressure sensors for sloshing experiment. Ocean Eng. 2015, 94, 199-212. [CrossRef]

11. Lu, Y.; Zhou, T.; Cheng, L.; Zhao, W.; Jiang, H. Dependence of critical filling level on excitation amplitude in a rectangular sloshing tank. Ocean Eng. 2018, 156, 500-511. [CrossRef]

12. Yu, L.; Xue, M.-A.; Zheng, J. Experimental study of vertical slat screens effects on reducing shallow water sloshing in a tank under horizontal excitation with a wide frequency range. Ocean Eng. 2019, 173, 131-141. [CrossRef]

13. Stoughton, R.S.; Arai, T. A modified Stewart platform manipulator with improved dexterity. IEEE Trans. Robot. Autom. 1993, 9, 166-173. [CrossRef]

14. Wang, S.-M.; Ehmann, K.F. Error model and accuracy analysis of a six-DOF Stewart platform. J. Manuf. Sci. Eng. 2002, 124, 286-295. [CrossRef]

15. Ding, B.; Cazzolato, B.S.; Stanley, R.M.; Grainger, S.; Costi, J.J. Stiffness analysis and control of a Stewart platform-based manipulator with decoupled sensor-actuator locations for ultrahigh accuracy positioning under large external loads. J. Dyn. Syst. Meas. Control 2014, 136. [CrossRef]

16. Eversafe Marine Engineering CO., LTD. Available online: www.eversafemarine.net/project-detail/1600tonnes-lpg-tank-lifting-project/ (accessed on 28 May 2020).

17. Hirt, C.W.; Nichols, B.D. Volume of Fluid (VOF) method for the Dynamics of free boundaries. J. Comput. Phys. 1981, 39, 201-225. [CrossRef]

18. Sussman, M.; Smereka, P.; Osher, S. A level set approach for computing solutions to incompressible two-phase flow. J. Comput. Phys. 1994, 114, 146-159. [CrossRef]

19. Monaghan, J.J. An introduction to SPH. Comput. Phys. Commun. 1988, 48, 89-96. [CrossRef]

20. Koshizuka, S.; Oka, Y. Moving-particle semi-implicit method for fragmentation of incompressible fluid. Nucl. Sci. Eng. 1996, 123, 421-434. [CrossRef]

21. Crespo, A.J.C.; Dominguez, J.M.; Rogers, B.D.; Gomez-Gesteira, M.; Longshaw, S.; Canelas, R.; Vacondio, R.; Barreiro, A.; Garcia-Feal, O. DualSPHysics: Open-source parallel CFD solver based on Smoothed Particle Hydrodynamics (SPH). Comput. Phys. Commun. 2015, 187, 204-216. [CrossRef]

22. FunctionBay. RecurDyn Manual, Version V9R2; FunctionBay: Gyeonggi-do, Korea, 2020.

23. Siemens. STAR-CCM+ User Guide, Version 15.02; Siemens: Munich, Germany, 2020.

24. Kim, D.H.; Kim, E.S.; Shin, S.-C.; Kwon, S.H. Sources of the measurement error of the impact pressure in sloshing experiments. J. Mar. Sci. Eng. 2019, 7, 207. [CrossRef]

25. Lamb, H. Hydrodynamics, 6th ed.; Cambridge University Press: Cambridge, UK, 1932.

26. Monaghan, J.J. Smoothed particle hydrodynamics. Annu. Rev. Astron. Astrophys. 1992, 30, 543-574. [CrossRef]

27. Wendland, H. Piecewise polynomial, positive definite and compactly supported radial functions of minimal degree. Adv. Comput. Math. 1995, 4, 389-396. [CrossRef]

28. Molteni, D.; Colagrossi, A. A simple procedure to improve the pressure evaluation in hydrodynamics context using the SPH. Comput. Phys. Commun. 2009, 180, 861-872. [CrossRef]

29. Crespo, A.J.C.; Gomez-Gesteira, M.; Dalymple, R.A. Boundary conditions generated by dynamic particles in SPH methods. CMC-Comput. Mater. Contin. 2007, 5, 173-184.

30. Grabcad Community. Available online: https:/grabcad.com/library/stewart-platform-mechanism (accessed on 1 March 2019).

31. Seyyedian Choobi, M.; Haghpanahi, M.; Sedighi, M. Investigation of the effect of clamping on residual stresses and distortions in butt-welded plates. Mech. Eng. 2010, 17, 387-394. 
32. Adami, S.; Hu, X.Y.; Adams, N.A. A generalized wall boundary condition for smoothed particle hydrodynamics. J. Comput. Phys. 2012, 231, 7057-7075. [CrossRef]

33. Fourtakas, G.; Dominguez, J.M.; Vacondio, R.; Rogers, B.D. Local Uniform Stencil (LUST) boundary condition for arbitrary 3-D boundaries in parallel smoothed particle hydrodynamics (SPH) models. Comput. Fluids 2019, 190, 346-361. [CrossRef]

(C) 2020 by the authors. Licensee MDPI, Basel, Switzerland. This article is an open access article distributed under the terms and conditions of the Creative Commons Attribution (CC BY) license (http://creativecommons.org/licenses/by/4.0/). 